\title{
Patients with familial biparental hydatidiform moles have normal methylation at imprinted genes
}

\author{
Osman El-Maarri ${ }^{1}$, Muhieddine Seoud ${ }^{2}$, Jean-Baptiste Rivière ${ }^{3}$, Johannes Oldenburg ${ }^{1,4}$, Jörn \\ Walter $^{5}$, Guy Rouleau ${ }^{3}$ and Rima Slim*,6
}

\begin{abstract}
${ }^{1}$ Institute of Experimental Haematology and Transfusion Medicine, Bonn, Germany; ${ }^{2}$ Department of Obstetrics and Gynecology, American University of Beirut, Beirut, Lebanon; ${ }^{3}$ Department of Medicine, Centre Hospitalier de l'Université de Montréal, Montreal, Canada; ${ }^{4}$ Institute of Transfusion Medicine and Immunohaematology, Frankfurt, Germany; ${ }^{5}$ Saarland University Biosciences Department of Genetics/Epigenetics, Saarbrücken, Germany; ${ }^{6}$ Department of Human Genetics and Obstetrics and Gynecology, McGill University Health Center, Montreal, Canada
\end{abstract}

In molar tissues from patients with recurrent biparental hydatidiform moles, we could previously demonstrate that differentially methylated regions (DMRs) of four imprinted genes are abnormally methylated on the maternal alleles. It remained unclear if this abnormal methylation originated de novo in the molar tissues or if it is even recognizable in the patient somatic tissues. To address this question, we investigated the DNA methylation of four imprinted genes in total blood from the two sister-patients. Here, we show that both patients retain normal methylation levels at the DMRs of the four genes in blood tissues. For two maternally expressed genes, we could use informative SNPs to follow the inheritance of the abnormally methylated maternal alleles in the molar tissues. We find that the transmitted abnormally methylated maternal alleles to the moles originated from the maternal grandmother and that the same alleles are not methylated in the patients. Our data suggest that the abnormal methylation in familial biparental molar tissues was acquired de novo in the patients'germline as a result of a false reprogramming or during the postzygotic development of the conceptuses that led to moles.

European Journal of Human Genetics (2005) 13, 486-490. doi:10.1038/sj.ejhg.5201353

Published online 12 January 2005

Keywords: hydatidiform moles; molar pregnancy; methylation analysis; SIRPH; imprinting

\section{Introduction}

Recurrent complete hydatidiform moles are a rare clinical entity in which molar tissues are diploids and have a biparental contribution to their genome (BiCHMs). These moles are undistinguishable, at both gross morphology and histopathology levels, from diploid androgenetic moles containing two sets of paternal chromosomes. Despite their diploid biparental genotype, the epigenotype

*Correspondence: Dr R Slim, Montreal General Hospital Research Institute, Room L12-132, 1650 Cedar Avenue, Montreal, Canada H3G 1A4, Tel: + 5149341934 ext 44519; Fax: + 514934 8265;

E-mail: rima.slim@muhc.mcgill.ca

Received 16 September 2004; revised 28 October 2004; accepted 11 November 2004 of BiCHMs is abnormal. Others and we demonstrated aberrant methylation and expression of imprinted, both maternally or paternally expressed, genes in BiCHMs. ${ }^{1-3}$ We showed that this aberrant methylation affects only the maternal alleles. ${ }^{3}$ To date, no studies has investigated if the methylation or expression of imprinted genes in the patients themselves is also affected, hence predisposing the patients to transmit such imprinting defects. This question is particularly interesting since patients with recurrent BiCHMs usually have miscarriages alternating with the moles and manifest during some of their molar pregnancies, complications and features of pre-eclampsia. Both recurrent pregnancy losses and pre-eclampsia are associated, in some cases, with physiological abnormalities 
such as folate deficiency and hyperhomocystemia. ${ }^{4-9}$ Folate and homocysteine are important components required for DNA and histone methylation. A defect in the methionine pathway in the two patients could be responsible or contribute to the molar phenotype and may manifest in an abnormal DNA methylation in their blood tissues.

We previously reported abnormal methylation at differentially methylated region (DMRs) of four imprinted genes in two molar tissues BiCHMs 9 and 16 from two sisters with recurrent BiCHMs, patients 4 and 6 from family MoLb1. ${ }^{3,10}$ The aim of the present study was to follow up this investigation by analyzing the DNA methylation status at the same DMRs, but in somatic tissues (blood) from the two patients. Furthermore, using SNPs in informative samples and at informative DMRs, we analyzed the grandparental transmission of the abnormal epigenotype to examine its potential association with a particular grandparental inheritance. Altogether, our data contribute to a better understanding of the developmental events occurring during biparental molar pregnancies and suggest that germline reprogramming or/and postzygotic defects are the major cause of BiCHMs.

\section{Results}

Methylation of four imprinted genes in patients with recurrent $\mathrm{BiCHMs}$

Methylation at SNRPN, PEG3, NESP55, and H19 DMRs was investigated on DNAs from total fresh blood of two patients with recurrent biparental moles, members 4 and 6 from family MoLb1, by two independent bisulfite methods as described previously. ${ }^{3}$ DNA was treated with sodium bisulfite, amplified by PCR, and the methylation was assessed by single-nucleotide primer extension (SNuPE) followed by separation with ion pair reverse-phase HPLC (SIRPH) ${ }^{11,12}$ and by cloning and sequencing. SIRPH analysis at the four DMRs was performed at two sites within each of the SNRPN, PEG3, and NESP55 DMRs, and at one site at the $H 19$ DMR as described previously. ${ }^{3}$ Our results on the four DMRs demonstrate a normal level of methylation, ranging from 35 to $45 \%$, in the two patients similar to the methylation level found in total blood from healthy normal subjects (Figure 1).

We also used cloning and sequencing to assess the DNA methylation of several adjacent CpG by sequencing individual clones from each of the four DMRs. The results at the DMRs without polymorphisms showed normal methylation pattern with some clones being completely methylated and others completely unmethylated (see Supplementary material). DMRs containing SNPs were at the NESP55 in patient 6 and at the H19 in both patients 6 and 4. Analysis of sequences from these DMRs demonstrated normal methylation pattern with only clones carrying the paternal alleles being methylated (Figure 2). These data demonstrate that the two patients with

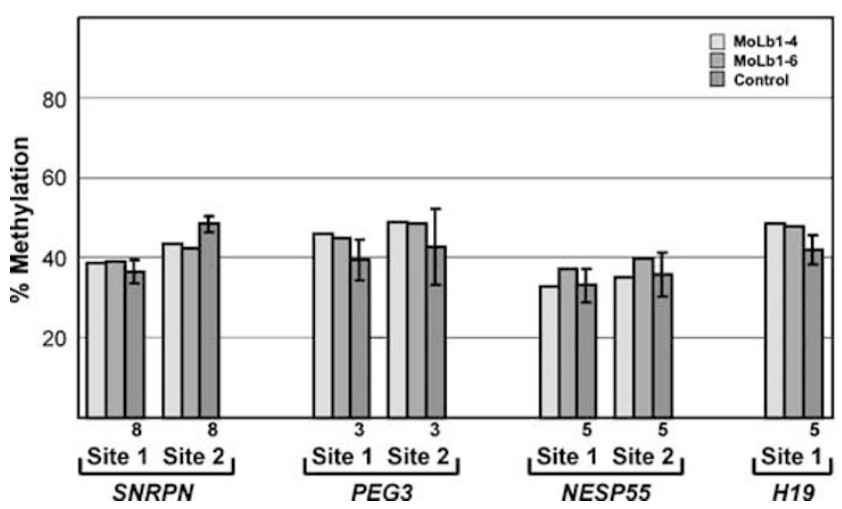

Figure 1 Quantitative methylation measurements by SIRPH analysis. Measurements were carried out at two sites at each of the SNRPN, PEG3, and NESP55 DMRs, and at one site at the H19 DMR. At each site, the first column from the left represents patient MoLb1-4, the second MoLb1-6 and the third is the average of measurements obtained on three to eight control DNA as indicated below each column. The different measurements represent independent samples from different subjects.

recurrent biparental moles have normal DNA methylation at the four analyzed imprinted genes.

Grandparental origin of the abnormally methylated maternal alleles in molar tissues

We previously demonstrated an abnormal methylation at the maternal SNRPN and NESP55 DMRs in BiCHMs 16 and 9, respectively. ${ }^{3}$ Using microsatellite markers in proximity of the SNRPN and NESP55 DMRs, cen-D15S1021-(450 kb)D15S128-(350 kb)-SNRPN DMR and cen-D20S164-(400 kb)NESP55 DMR-(400 kb)-D20S171, respectively, we established the haplotypes carrying the abnormally methylated alleles. Haplotype analysis shows that the abnormally unmethylated maternal SNRPN in BiCHM 16, with C at position 144772 (GenBank Accession no. AC009696), is inherited from the maternal grandmother (haplotype 4-3C) (Figure 3a). In BiCHM 9, the abnormally methylated maternal NESP55 allele, with A at position 106675 (GenBank accession no. AL132655), is inherited from the maternal grandmother (haplotype 1-A-1) (Figure 3). We note that the healthy daughter of patient MoLb1-4, with normal methylation level and pattern, has inherited the same grandparental haplotype (1-A-1).

\section{Discussion}

To investigate whether two sister-patients with BiCHMs have abnormal DNA methylation at imprinted genes in their somatic tissues, we analyzed four DMRs that we previously showed are abnormally methylated/unmethylated on the maternal alleles in their molar tissues. ${ }^{3}$ Using SIRPH analysis on blood DNA from the two patients, we demonstrate normal methylation levels at the four genes (Figure 1). Cloning and sequencing data on DMRs without 


\section{Maternally Expressed Genes}
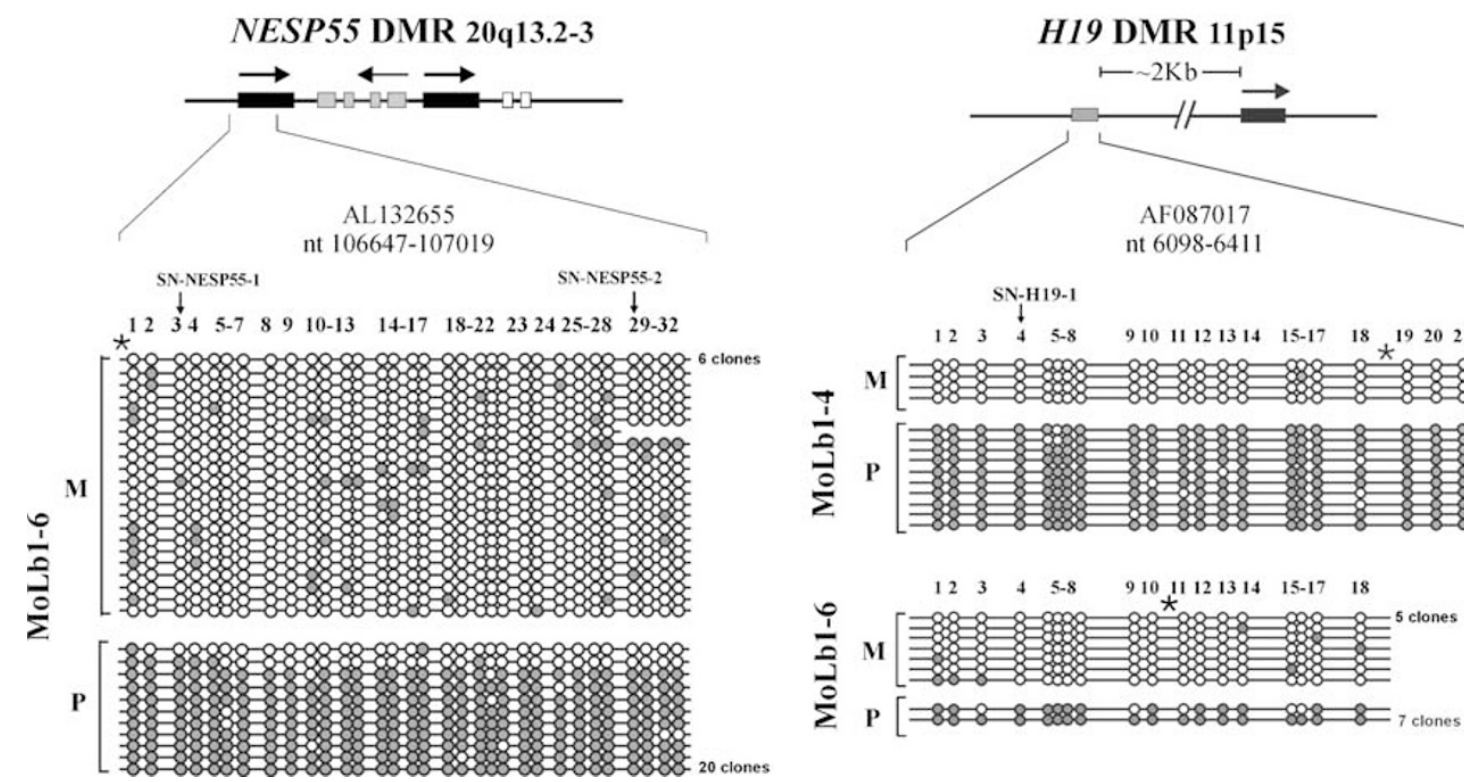

Figure 2 Summary of the methylation analysis from isolated sequenced clones at the NESP55 and H19 DMRs. The studied region relative to each gene is shown under the corresponding name. Each individual PCR clone is represented by a line; unmethylated and methylated CpG sites are represented by open and filled circles, respectively. The number of clones with identical methylation pattern is indicated on the right side of one representative clone. Vertical arrows indicate the sites studied by the SIRPH analysis. M: maternal; P: paternal. The position of the polymorphisms that distinguish the parental alleles is indicated by '*'. These are at position 106675 in AL132655 for NESP55 and at positions 6225 (MoLb1-6) and 6336 (MoLb1-4) in AF087017 for H19.

polymorphisms are compatible with normal methylation pattern (Supplementary material). With the use of available SNPs, we demonstrate a normal methylation pattern at NESP55 in one patient, MoLb1-6, and at H19 in both of them (Figure 2).

We previously traced the inheritance of the abnormally methylated maternal $\mathrm{H} 19$ alleles in molar tissues and found that they are inherited from the maternal grandfather to BiCHM9 and from the maternal grandmother to BiCHM16. Now, we show that the two patients have normal methylation level and pattern at H19, with only their paternal alleles being methylated (Figure 2). The presence of abnormal methylation marks in BiCHM16 on a nonmethylated allele in the patient's blood indicates that the abnormal methylation was acquired in the germline of the patient or during the postzygotic development of the BiCHM16. This suggests that the abnormal epigenotype in the BiCHM16 did not originate in the mothers' somatic tissues as a result of a physiological impairment, for instance, in the methylation pathway. Using microsatellite flanking the NESP55 DMR, we show that the abnormally methylated allele in BiCHM9 is also inherited from the maternal grandmother (Figure 3b). The patient, MoLb1-4, has normal level of methylation in her total blood; beside her reproductive problem, she has a normal phenotype and is in good health. Consequently, the patient is expected to have normal methylation pattern with methylation only on her paternal NESP55 DMR. This demonstrates, again, at a second locus, NESP55, and in a second mole, BiCHM9, the acquisition of new methylation marks on a nonmethylated allele transmitted by the patient. At the SNRPN, we show that the abnormally unmethylated maternal allele in BiCHM16 is inherited from the maternal grandmother (Figure 3a). The patient, MoLb1-6, has normal methylation level in her blood and is expected to have methylation marks only on her maternal allele. Therefore, the abnormal absence of methylation on the SNRPN maternal allele in BiCHM16 could result from an incomplete erasure, an incomplete reprogramming, or abnormal postzygotic maintenance of the correctly established marks.

Of all the studies that have addressed the methylation or expression of imprinted genes in familial molar tissues, none of them allows reaching a conclusion as to whether the abnormal methylation occurred in the maternal germline or during the postzygotic development for the following reasons. First, all these studies were performed on molar tissues at 6-14 weeks of gestation in which several changes could have occurred since fertilization. Consequently, the abnormal methylation observed in these tissues may not reflect exactly the methylation marks of the parental gametes. Second, although abnormal unmethylation was reported at several imprinted, paternally expressed genes with primary imprints (known to be established in the germline), but abnormal methylation 
a

Paternally expressed $S N R P N$

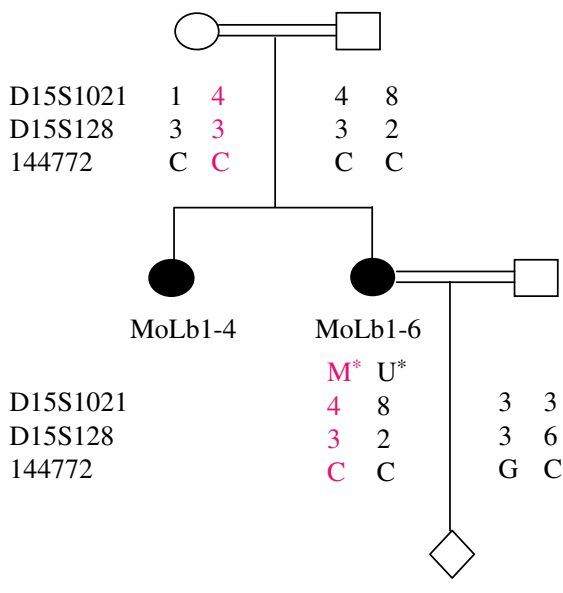

BiCHM 16

D15S1021
D15S128
144772

D15S 1021
D15S 128

144772 b Maternally expressed NESP55

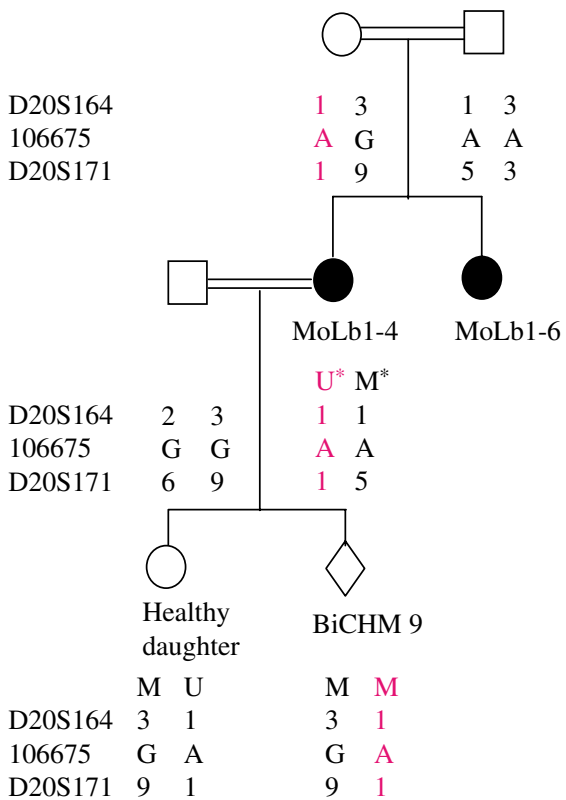

Figure 3 Segregation of the grandparental haplotypes at SNRPN and NESP55 to BiCHMs. Haplotypes were constructed assuming minimal number of recombinations. The order of the markers is given from the centromere (top) to the telomere (bottom) according to the UCSC map (http:// genome.ucsc.edu). The positions of the SNPs at the SNRPN and NESP55 DMRs are given in GenBank Accession sequences AC009696 and AL132655, respectively. The haplotypes carrying the abnormally methylated alleles in molar tissues are in red, ' $U$ ' indicates haplotypes carrying the unmethylated allele and ' $\mathrm{M}$ ' the methylated one based on the presence of informative SNPs; an asterisk '*' is added to haplotypes without informative SNPs in the patients and whose methylation status is inferred based on the normal level of methylation obtained by SIRPH and on the normal phenotype of the patients.

marks were also shown at NESP55, ${ }^{1,3}$ with secondary imprints (known to be established postzygotically around the blastocyst stage). Third, molar pregnancies are benign tumors of the trophoblast and several studies have shown gain or loss of DNA methylation at imprinted genes, including PEG3 and $H 19$ (extensively studied), in a variety of tumors such as glioma, Wilms' tumor, lung carcinoma, Müllerian tumor of the uterus, hepatoblastoma, and colorectal cancer. ${ }^{13-21}$ In colorectal cancer and Wilms' tumors, a gain of methylation on the maternal H19 DMR was shown. ${ }^{13,14,16,20,21}$ Therefore, it would be expected that at least some of the methylation changes observed in biparental molar tissues (gain or loss) have occurred during molar development and are specific for these trophoblastic tumors. This would explain the deregulation of the same genes in the same way in most analyzed biparental moles, familial and sporadic. ${ }^{2,3}$

An intriguing observation in biparental moles is the loss of maternal methylation imprints on most of the analyzed paternally expressed genes while paternal methylation imprints were maintained on two analyzed maternally expressed genes. This led Judson et $a l^{1}$ to suggest that a 'maternal' germline defect in establishing the maternal imprints underlies familial BiCHMs. Although this hypothesis is attractive and plausible, it may not be the only explanation. Another explanation that may underlie the different behavior between maternal and paternal imprints would be due to their different properties and is supported by several observations in BWS and PWS syndromes. In case of the BiCHMs, the labile nature of maternal imprints in placental tissues during early development may explain the loss of maternal imprints. ${ }^{22}$

Finally, the abnormal methylation patterns on the maternal alleles of maternally or paternally expressed genes in biparental molar tissues may have not occurred at all DMRs at the same time, but resulted from several events that altogether led to the molar phenotype.

\section{Acknowledgements}

We thank the patients for their cooperation and Judith Schwalbach for technical support. RS is supported by the 'Fonds de Recherche en Sante' $d u$ Québec' and by an operating grant from the CIHR (MOP-67179).

\section{References}

1 Judson H, Hayward BE, Sheridan E, Bonthron DT: A global disorder of imprinting in the human female germ line. Nature 2002; 416: 539-542.

2 Fisher RA, Hodges MD, Rees HC et al: The maternally transcribed gene p57(KIP2) (CDNK1C) is abnormally expressed in both androgenetic and biparental complete hydatidiform moles. Hum Mol Genet 2002; 11: 3267-3272. 
3 El-Maarri O, Seoud M, Coullin P et al: Maternal alleles acquiring paternal methylation patterns in biparental complete hydatidiform moles. Hum Mol Genet 2003; 12: 1405-1413.

4 Nelen WL, Blom HJ, Steegers EA, den Heijer M, Eskes TK: Hyperhomocysteinemia and recurrent early pregnancy loss: a meta-analysis. Fertil Steril 2000; 74: 1196-1199.

5 Nelen WL, Blom HJ, Steegers EA, den Heijer M, Thomas CM, Eskes TK: Homocysteine and folate levels as risk factors for recurrent early pregnancy loss. Obstet Gynecol 2000; 95: 519-524.

6 Nelen WL, Bulten J, Steegers EA, Blom HJ, Hanselaar AG, Eskes TK: Maternal homocysteine and chorionic vascularization in recurrent early pregnancy loss. Hum Reprod 2000; 15: 954-960.

7 Barro C, Ducros V, Bonaz B et al: Homocysteinemia and recurrent fetal losses: description of three cases. Ann Biol Clin (Paris) 2002; 60: $325-326$.

8 Kumar KS, Govindaiah V, Naushad SE, Devi RR, Jyothy A: Plasma homocysteine levels correlated to interactions between folate status and methylene tetrahydrofolate reductase gene mutation in women with unexplained recurrent pregnancy loss. $J$ Obstet Gynaecol 2003; 23: 55-58.

9 Reznikoff-Etievant MF, Zittoun J, Vaylet C, Pernet P, Milliez J: Low vitamin B12 level as a risk factor for very early recurrent abortion. Obstet Gynecol 2002; 104: 156-159.

10 Moglabey YB, Kircheisen R, Seoud M, El Mogharbel N, Van den Veyver I, Slim R: Genetic mapping of a maternal locus responsible for familial hydatidiform moles. Hum Mol Genet 1999; 8: $667-671$.

11 El-Maarri O, Herbiniaux U, Walter J, Oldenburg J: A rapid, quantitative, non-radioactive bisulfite-SNuPE-IP RP HPLC assay for methylation analysis at specific CpG sites. Nucleic Acids Res 2002; 30: e25.

12 El-Maarri O: SIRPH analysis: SNuPE with IP-RP-HPLC for quantitative measurements of DNA methylation at specific CpG sites. Methods Mol Biol 2004; 287: 195-206.
13 Steenman MJ, Rainier S, Dobry CJ et al: Loss of imprinting of IGF2 is linked to reduced expression and abnormal methylation of H19 in Wilms' tumor. Nat Genet 1994; 7: 433-439.

14 Moulton T, Crenshaw T, Hao Y et al: Epigenetic lesions at the H19 locus in Wilms' tumour patients. Nat Genet 1994; 7: 440-447.

15 Kondo M, Suzuki H, Ueda R et al: Frequent loss of imprinting of the $\mathrm{H} 19$ gene is often associated with its overexpression in human lung cancers. Oncogene 1995; 10: 1193-1198.

16 Taniguchi T, Sullivan MJ, Ogawa O, Reeve AE: Epigenetic changes encompassing the IGF2/H19 locus associated with relaxation of IGF2 imprinting and silencing of H19 in Wilms tumor. Proc Natl Acad Sci USA 1995; 93: 2159-2163.

17 Hashimoto K, Azuma C, Tokugawa Yet al: Loss of H19 imprinting and up-regulation of $\mathrm{H} 19$ and SNRPN in a case with malignant mixed Mullerian tumor of the uterus. Hum Pathol 1997; 28: $862-865$

18 Li X, Kigner P, Sandstedt B et al: Promoter-specific methylation and expression alterations of Igf2 and $\mathrm{H} 19$ are involved in human hepatoblastoma. Int J Cancer 1998; 75: 176-180.

19 Maegawa S, Yoshioka H, Itaba N et al: Epigenetic silencing of PEG3 gene expression in human glioma cell lines. Mol Carcinogen 2001; 31: 1-9.

20 Cui H, Niemitz EL, Ravenel JD et al: Loss of imprinting of insulinlike growth factor-II in Wilms' tumor commonly involves altered methylation but not mutation of CTCF or its binding site. Cancer Res 2001; 61: 4947-4950.

21 Nakagawa H, Chadwick RB, Peltomäki P, Plass C, Nakamura Y, de la Chapelle A: Loss of imprinting of the insulin-like growth factor II gene occurs by biallelic methylation in a core region of H19associated CTCF-binding sites on colorectal cancer. Proc Natl Acad Sci USA 2001; 98: 591-596.

22 Mann MR, Lee SS, Doherty AS et al: Selective loss of imprinting in the placenta following preimplantation development in culture. Development 2004; 131: 3727-3735.

Supplementary Information accompanies the paper on European Journal of Human Genetics website (http://www.nature.com/ejhg) 